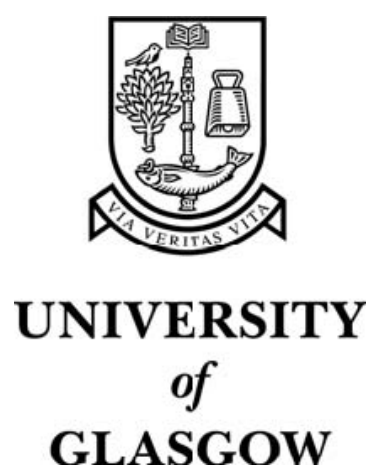

Potts, H.E. and Khan, J.I. and Diver, D.A. (2007) Small scale energy release driven by supergranular flows on the quiet Sun. Solar Physics 245(1):pp. 55-68.

http://eprints.gla.ac.uk/3753/ 


\title{
Small scale energy release driven by supergranular flows on the quiet Sun
}

\author{
H. E. Potts, J. I. Khan and D.A. Diver \\ Department of Physics and Astronomy, University of Glasgow, Glasgow, UK
}

\begin{abstract}
In this article we present data and modelling for the quiet Sun that strongly suggest a ubiquitous small-scale atmospheric heating mechanism that is driven solely by converging supergranular flows.

A possible energy source for such events is the power transfer to the plasma via the work done on the magnetic field by photospheric convective flows, which exert drag of the footpoints of magnetic structures. In this paper we present evidence of small scale energy release events driven directly by the hydrodynamic forces that act on the magnetic elements in the photosphere, as a result of supergranular scale flows. We show strong spatial and temporal correlation between quiet Sun soft X-ray emission (from Yohkoh SXT) and $S O H O$ MDI-derived flux removal events driven by deduced photospheric flows.

We also present a simple model of heating generated by flux submergence, based on particle acceleration by converging magnetic mirrors.

In the near future, high resolution soft X-ray images from XRT on the Hinode satellite will allow definitive, quantitative verification of our results.
\end{abstract}

\section{Introduction and motivation}

Solar coronal heating is one of the outstanding unresolved solar physics problems, with no clear consensus as to the definitive cause. There are a huge range of known energy release mechanisms on the Sun (see for example Erdélyi (2005) and references therein), many of which have been implicated for coronal heating. At the largest scales there are the relatively rare events such as large flares and CMEs, which are considered too infrequent to contribute significantly to coronal heating. Moving down in scale the events become much more frequent, such as micro- and nano-flares, soft Xray bright points and blinkers. There are also continuous processes such as acoustic and MHD wave heating by various mechanisms. As the spatial and temporal resolution of observing instruments grows, we see ever increasing numbers of smaller events, and there have been studies that suggest these small events may significantly contribute to the heating budget (Parnell and Jupp, 2000; Hudson, 1991).

Small scale heating has long been associated with regions of flux cancellation (Webb et al., 1993); these have normally been interpreted as small-scale reconnection events (Priest, Parnell, and Martin, 1994). However, there is evidence that the flux cancellation observed may not be the result of reconnection (Chae et al., 2002), but be due to a flux submergence process

(C) 2007 Springer Science + Business Media. Printed in the USA. 
(Harvey et al., 1999; Chae, Moon, and Pevtsov, 2004), where magnetic loops retract below the solar surface.

In this paper we look at a driving mechanism for small scale energy release by flux submergence: the work done on the magnetic field by hydrodynamic dragging of magnetic footpoints in the large scale photospheric flows. The research presented here, using detailed observations and data reduction, shows temporal and spatial correlation between regions of converging photospheric flow in the quiet Sun, the motion of the entrained magnetic field and the resultant small scale energy releases. We present a simple theoretical model that shows that heating will occur as a flux loop submerges beneath the chromosphere, without requiring magnetic reconnection.

We hope to extend this treatment to a more quantitative study of the energy transfer when the greatly improved quality of data from the new Hinode satellite becomes available.

The analysis is presented in sections as follows: Section 2 demonstrates how fluid and magnetic element motion is deduced from the datasets, and shows close correlation between the two. Section 3 shows how SXT bright points are spatially and temporally correlated with the flux removal or disappearance, suggesting that the two are causally linked. Section 4 offers a simple model framework that offers a possible explanation for small-scale heating that is consistent with these observations. Section 5 is a concluding discussion.

\section{Photospheric flow and magnetic element motion}

It is well known that the magnetic elements are advected to supergranular boundaries, see for example Simon et al., (1988), Hagenaar et al., (1999) and Lisle, De Rosa, and Toomre (2000). This concentrates the magnetic flux at the supergranular boundaries, one result of which is the chromospheric network. In this section we compare the motion of the photospheric plasma (derived from tracking the motion of granules) to that of the smallest magnetic elements that we can track from high resolution MDI magnetograms, in order to quantify the relation between the two motions. The results from this are used in Section 3 in order to calculate the rate of flux removal.

The source of the data used in this section is a 33 hour set (2000 images at 1 minute cadence) of high resolution MDI continuum and line-of-sight magnetogram images observed in a quiet region near disk centre, starting on 15 January 1997. This data set was chosen as it gives the largest continuously observed area of the solar surface at high resolution that has ever been obtained from MDI. The data was rigidly derotated at 0.243 pixels $/ \mathrm{min}$ before analysis, giving a $480 \times 1024$ pixels $\times 2000$ frames $(4.8 \times 10.3$ arcmin $\times 33$ 
hours) data cube, with mean velocity close to zero. The residual differential rotation was removed during later processing.

There are three main stages to the data analysis: measuring the photospheric flow velocities, analysing the cell structure in the velocities, and tracking the motion of magnetic elements. These steps are given in detail in the following subsections.

\subsection{Surface Flows and Supergranulation Structure}

The photospheric flows were obtained by tracking the barely resolved granulation patterns from high resolution MDI continuum data. The data were first spatially filtered to remove $p$-mode oscillations (which dominate the raw data) and rigidly derotated at 0.243 pixels/minute which closely matches the mean fluid rotation speed for the data over the latitude range. The filtering and derotation were performed in Fourier space for speed and to avoid interpolation errors (Potts, Barrett, and Diver, 2003). The filtered, derotated data were then processed to yield the photospheric velocity fields using the Balltrack method; full details of this technique are given in Potts, Barrett, and Diver (2004) and are not repeated here.

The supergranulation pattern, and its time evolution, were deduced from the high resolution photospheric velocity data described above. The method we used is described in detail in another publication (Potts and Diver, 2007), and so we shall only give a brief overview. The algorithm is a development of the watershed basin algorithm, commonly used by hydrographers for the analysis of river basins, and described in the solar context by DeRosa and Toomre (2004). The observed velocity field is first time-reversed, so that the flow is reversed. A regular array of tracking particles, distributed across the entire region of interest, is launched into the reversed flow field, causing particles to migrate under the convergent flow conditions to different cell outflow centres. The boundaries of cells can be deduced by considering the rate of change of a tracking particle's final position compared to its initial one. This gives an exceptionally low noise measurement of the local divergence of the flow, as it uses a path integral of the flow, rather than the conventional approach of differentiating the data to obtain the divergence field. The outflow centre for each cell is also automatically obtained with high accuracy by the points where the tracking particles cluster. The results of this method can be seen in the bottom right graph in Figure 1 where the lanes are shown by the black lines, overlaid on an image of the magnetic flux density.

\subsection{Magnetic Element tracking}

We are interested in tracking the smallest possible magnetic elements, at the noise and spatial resolution limit of high resolution MDI magnetograms. 
Tracking these small elements is difficult, as in a single frame of data they are hard to distinguish from noise. In order to track them we use a targeted, multi-frame version of the local correlation tracking algorithm. This method exploits the fact that the motion of small magnetic elements tends to be linear, when observed at this spatial resolution over periods of around an hour. First the data are cleaned and binned into 10 minute cadence images, in order to reduce the noise on the data. Possible magnetic elements are then identified from each binned frame by means of a multi-level contouring algorithm. A circular subimage, with three times the diameter of the identified element is then extracted. A cumulative correlation between this subimage and the surrounding \pm 3 frames is then calculated, for all possible $x$ and $y$ velocities of the element. The $x$ and $y$ velocity that gives the best correlation is selected, and if the cumulative correlation is high enough the element's position (centroid), velocity and total flux is stored. The strength of this method is that it effectively averages 70 frames of data with the resultant 8 -fold reduction in noise, but without blurring moving features. It automatically gives a high immunity to false detections due to noise, as the element must appear with a consistent shape and velocity in all 7 frames, and also gives an estimate of the element velocity. We obtained reliable tracks from elements with an area as small as 4 MDI pixels (only $1 \mathrm{Mm}^{2}$ ), right at the diffraction limit of MDI.

The individual features obtained were then assembled into tracks, using the centroid, velocity estimate and total flux data and taking into account the splitting and merging of elements.

Using this method over the full 33 hour data set we identified the tracks of 3300 magnetic elements that did not merge or branch and which were able to be tracked for a minimum of 3 hours continuously, with no step changes in their total flux. This yielded around 16000 separate velocity and position measurements. A small subset of some of the tracks observed is shown in the top-left graph in Figure 1.

\subsection{Results}

Figure 1 shows a qualitative view of the main results, with a quantatative analysis in Figure 2. The bottom-right image shows a snapshot of the derived supergranular pattern, overlaid onto an image of the magnetic flux density. The magnified inset shows an example of the elements selected for tracking. The visible pixels in the magnified image indicate the high sensitivity of the method to small elements - some are only 2 pixels (around $1 \mathrm{Mm}$ ) wide. Note the clear concentration of the flux at the supergranular boundaries and vertices. A movie of this that strikingly shows the advection of the elements and the evolution of the supergranular pattern may be found in the electronic supplement to this paper (supergran_mag_overlay.avi) and on 


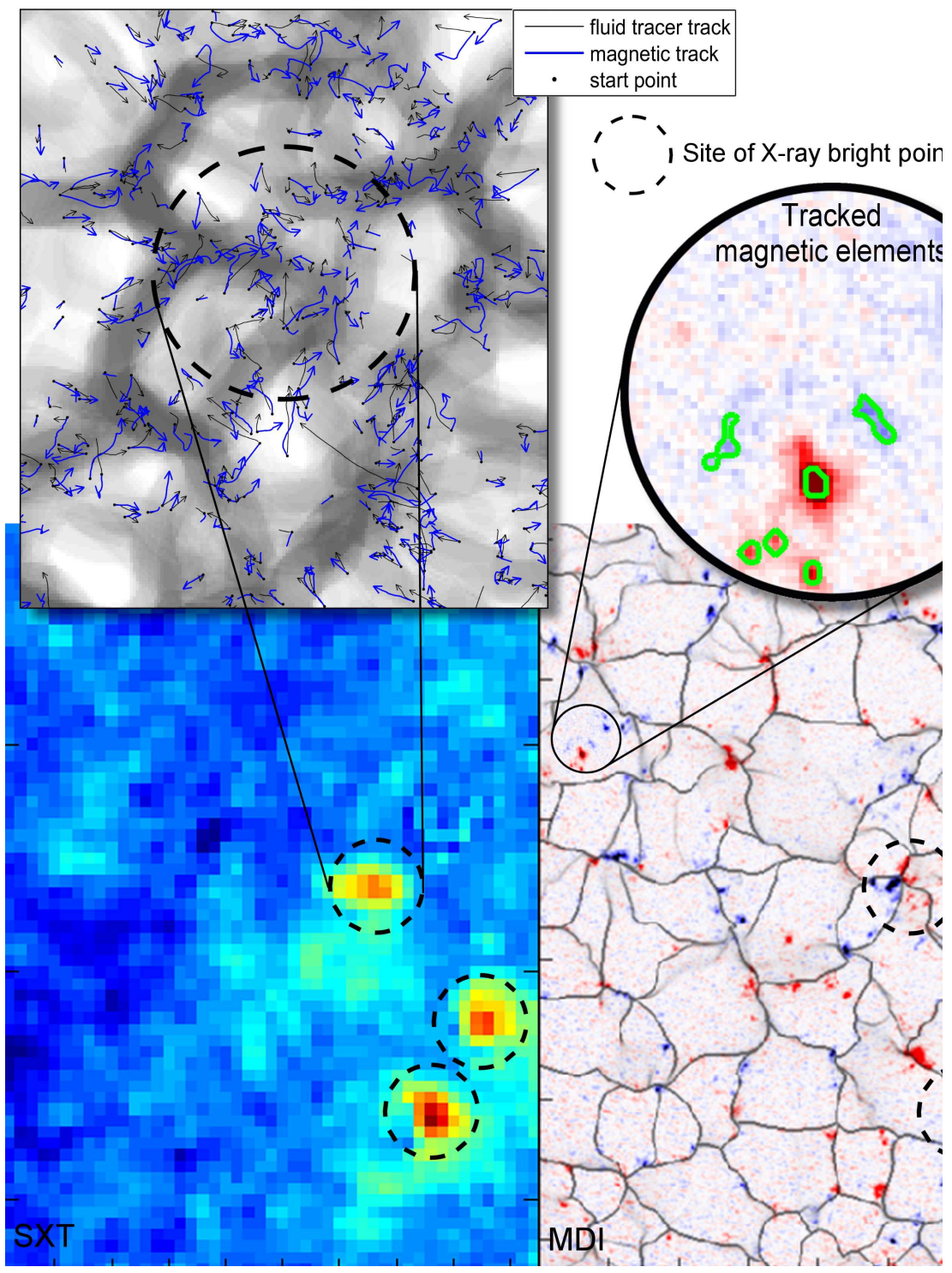

Figure 1. X-ray bright points detected with SXT (bottom left) compared with the state of the magnetic field (bottom right, red and blue for positive and negative polarity respectively) and the supergranulation cells (gray lines). Note the high concentration of magnetic flux at the supergranular boundaries, shown more clearly in Figure 2. A movie of this that strikingly shows the advection of the elements and the evolution of the supergranular pattern may be found in the electronic supplement to this paper (supergran_mag_overlay.avi) and on the web in Potts (2007). The zoomed-in section at the top left shows the tracks of magnetic elements over 33 hours compared with the motion of fluid tracer particles over the same time span. Notice the general flow towards the area of energy release. The shading in the background represents the average position over the full time period of the supergranular lanes. For clarity only a fraction of the tracked elements are shown. The circular enlarged region at the top right shows the magnetic elements selected for tracking. The SXT and MDI images are $4 \times 5$ arcmin. 

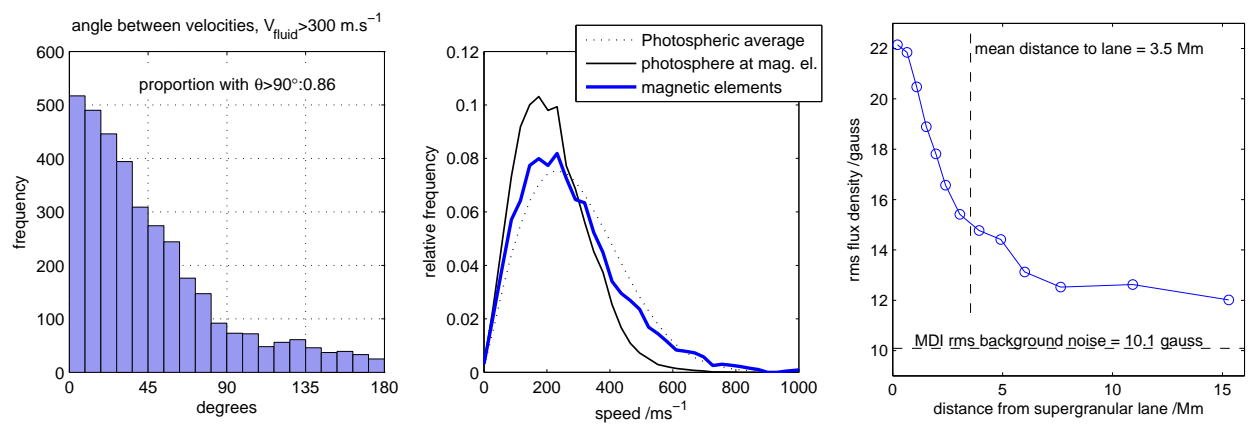

Figure 2. The left-hand figure shows the angle between the motion of magnetic elements, calculated over a 3 hour period, and the motion of a massless tracking particle that is advected by the photospheric flow over the same period. The centre figure shows speed distribution of the magnetic elements (solid thick) and the photospheric flow, globally (dotted) and sampled at the position of the magnetic elements (solid thin). The right-hand figure is a graph of the rms flux density versus the distance from the nearest supergranular lane for each point on the dataset, showing the concentration of flux at the supergranular lanes.

the web in Potts (2007). This concentration is quantified in the right-hand graph of Figure 2, discussed in more detail below. The top-left image in Figure 1 shows a comparison of the magnetic element motion to that of massless tracer particles that track the flow as it evolves over the 33 hour data window. The subarea chosen is in the vicinity of an SXT bright point, indicated in all plots by the dashed circles. The background dark shading indicates the average position of the supergranular lanes over the entire period. The correlation between the motions is clear: the directions of the element motions are confined to a small angular spread about the fluid flow direction, and the magnitudes of both motions are very similar. This is particularly true in the areas away from the supergranular lanes. A more quantitative analysis is shown in Figure 2. The right-hand plot in Figure 2 shows the rms magnetic field as a function of the distance to the nearest supergranular lane, from a 3 hour segment of data. In the quiet Sun, MDI magnetograms are dominated by noise, so to work out the enhancement of the flux density at the lane boundaries requires a measurement of the random noise in the magnetogram data. From the published SOHO MDI observables (Scherrer et al., 1995) the single measurement $1 \sigma$ noise on MDI magnetograms is 20 gauss. The rms flux densities that we measure here are as low as 12 gauss, showing that the noise in quiet Sun conditions must be less than this. The probable reason for this is that the error signal associated with strong flows and strong magnetic fields is smaller in the quiet Sun conditions (Lui and Norton, SOI Technical note 01-144). In order to estimate the noise amplitude we look at the difference between two consecutive frames. On this timescale (1 minute) the magnetic field at the resolution of MDI does 
not change significantly, so the residual gives a measure of the uncorrelated noise between the frames. Using this method we get a single pixel noise value of 10.1 gauss, shown in the right hand plot. The field enhancement between the supergranular lanes and the cell centres, taking into account the background noise, is around a factor of 5 . If just the pixels where the field is greater than 50 gauss are considered, then $80 \%$ of them lie within $2.1 \mathrm{Mm}$ of a supergranular lane, closer than the resolution of the velocity data, indicating that the downflow regions are very narrow, coherent and well defined, and confirming the high accuracy of the lane calculating algorithm. The left-hand plot of Figure 2 shows the relation between the angle of motion of magnetic elements averaged over 3 hours with that of the flow underlying them over the same period. Only elements where the underlying flow was faster than $300 \mathrm{~m} \mathrm{~s}^{-1}$ were selected, as small amounts of noise in the motion can result in a large angular error for low speed flows. As can be seen there is a strong alignment between the directions of motion, with $86 \%$ of the elements moving in the same direction as the flow. Any spurious correlation caused by the differential rotation of the Sun was removed by fitting a differential rotation profile to the velocity data ensuring that the average longitudinal motion of the velocity field was zero at all latitudes. This correction was also applied to the magnetic element velocities. The centre image shows a comparison of the distribution of the speeds of magnetic elements, with the distribution of the speed of the photospheric flow under the elements, and for comparison the distribution of flow speeds over the whole photospheric region. The results we obtain are very similar to those obtained by (Lisle, De Rosa, and Toomre, 2000) from full disk MDI data, with a modal velocity $220 \mathrm{~m} \mathrm{~s}^{-1}$ for the magnetic elements and a slightly lower modal velocity of $200 \mathrm{~m} \mathrm{~s}^{-1}$ for the photospheric flow, evaluated at the element positions. Note that this is a lower modal speed than that of the photosphere as a whole (dotted line), due to the concentration of the magnetic elements at the relatively slow moving supergranular boundaries. The speeds we obtain are significantly lower than those obtained from high cadence (20 s), very high resolution $(350 \mathrm{~km})$ ground based measurements of magnetic bright points (Berger et al., 1998), which yielded modal speeds of around $300 \mathrm{~m} \mathrm{~s}^{-1}$, and a much higher mean speed of nearly $1500 \mathrm{~m} \mathrm{~s}^{-1}$. The reason for this is that the high resolution of the ground based data allowed the small random motions of much smaller elements to be captured, but we can only resolve the larger scale drift velocity. Our restriction on the lifetime of the elements being a minimum of 3 hours also reduces the observed speed, as small, short lived elements have been observed to move faster (Lisle, De Rosa, and Toomre, 2000).

Key Result: The conclusion to this section is that the motion of the magnetic elements is very strongly correlated with the motion of the photo- 



Figure 3. An illustration of the method used to calculate the the rate of flux loss. First a circular region is defined (left hand diagram) and the magnetic flux within it calculated. The boundary of the region is then allowed to advect with the photospheric flow, shown by the black arrows. As the motion of small magnetic elements largely follows the photospheric flow (see Section 2 ) this ensures that no new flux crosses the boundary. Any changes in the flux contained within the loop are therefore due to flux creation or destruction in the area. The time delay between the two frames in this case is 2 hours, in order to show clearly the method. For the detailed analysis a time step of 10 minutes was used.

spheric flow they are embedded in. This is used in the following section for the calculation of the flux removal rate.

\section{Flux removal and SXT bright points}

The previous section describes a method to track many small magnetic elements. In principle it should be possible use these results to find areas where positive and negative magnetic field are disappearing together. Unfortunately, although we can track many elements, we can not track all of them, so distinguishing between physical flux removal and merely failing to track magnetic elements is not possible. Instead we use the result that the average motion of the magnetic elements is strongly correlated to the photospheric flow, and use the flow as a measure of the element advection.

In the first sub-section we describe the algorithm for detecting the flow removal regions, following that we describe detection of small X-ray bright points from Yohkoh SXT data. We then show some examples of flux removal, which have temporal and spatially aligned SXT bright points. 


\subsection{Locating FLUX REMOVAL REgions}

If we consider the change in the total magnetic flux at a small area of the photosphere there are two contributions:(i) the effect of the advection of flux into and out of the region, and (ii) flux creation and destruction within the region. In order to measure the rate of flux submergence or removal it is necessary to separate these two effects.

To do this we use the observation made in Section 2 that the motion of the small magnetic elements is strongly correlated with the photospheric flow, and the speeds of motion are very similar. If we define a circular area on the photosphere (left hand panel in Figure 3) and make the boundary of that region move as time progresses such that no magnetic flux crosses it, the change in total flux within that boundary is only due to flux generation or destruction. We move the boundary by moving the points on its perimeter at the same velocity $\mathbf{V}_{p}$ as the radial component of the photospheric velocity field:

$$
\mathbf{V}_{p}=\hat{\mathbf{r}}\left(\mathbf{V}_{f} \cdot \hat{\mathbf{r}}\right)
$$

where $\mathbf{V}_{f}$ is the photospheric velocity evaluated at the boundary point and $\hat{\mathbf{r}}$ is the radial unit vector from the centre of the circle to the perimeter point. To look specifically for the signature of submerging loops or local reconnection, we measure the simultaneous change in both positive and negative flux, $\beta$, at circles centred at each point, for frames separated by 10 minutes.

Define $\Delta \phi_{ \pm}=\left|\phi_{ \pm, f}\right|-\left|\phi_{ \pm, i}\right|$ as the absolute change in positive $(+)$ and negative (-) flux in the area defined by the circles centred at each point, for the initial (i) and final (f) frames which are separated by $\Delta t=10$ minutes. We can define $\beta$ as a useful measure of the simultaneous flux changes as follows:

$$
\beta=\frac{1}{\mathcal{A} \Delta t}\left[\frac{\Delta \phi_{+}}{\left|\Delta \phi_{+}\right|}+\frac{\Delta \phi_{-}}{\left|\Delta \phi_{-}\right|}\right] \times \min \left(\left|\Delta \phi_{+}\right|,\left|\Delta \phi_{-}\right|\right)
$$

where $\mathcal{A}$ is the fractional (non-dimensional) change in area of the region, and the function 'min' is used to select only those changes which can plausibly be considered as simultaneous in both flux components. The reason for including the area term is that in quiet Sun areas the MDI data has significant noise, the absolute sum of which over the region is proportional to the area of the region. Including this term will slightly reduce the signal in areas of true magnetic removal (typically by around $5 \%$ when using a 10 minute time step), but completely remove any spurious signals from areas that have a significant area change, but no flux change. The quantity $\beta$ is therefore an expression of the local rate of flux removal, averaged over the circle, with units of $\mathrm{Wb} \mathrm{s}^{-1}$. A map of the spatial variation of $\beta$ is built up by applying this algorithm at each surface point. The evolution of $\beta$ for 
selected regions is shown in Figure 4, and the spatial variation over small regions can be seen in Figure 5.

\subsection{Cleaning and Preparation of SXT Data}

The SXT data available for this region was in the form of half resolution and quarter resolution images $(256 \times 256$ pixels $)$ taken through a selection of filters. We did not find any suitable MDI quiet Sun data sets where SXT was running in its partial frame mode, overlapping the MDI high resolution field of view. Only the longest exposure images with the thinnest filter had high enough sensitivity to see any detail in the quiet Sun regions. After selecting the suitable frames we are left with 47 quiet Sun images at quarter resolution and 7 at half resolution.

The raw images from SXT over the quiet Sun are dominated by CCD flat field noise/ dark frame noise. The features for which we are searching in the SXT data are on the limit of its noise threshold, so careful processing to remove pixel artifacts was required. The standard Solarsoft image processing packages left substantial artifacts in the data, mostly due to dark frame errors, and so we used a more sophisticated processing technique in order to optimise the information content.

First, the permanently bright (hot) pixels were identified and removed. Then the subset of frames that were free from proton impacts were identified. The main source of image artifacts from SXT for quiet Sun images is the pixel-to-pixel dark current variation. A good measure of this in the quiet Sun is the standard deviation of the spatial discrete Laplacian for the time average of the data. This quantity was minimised by iteratively removing the dark frame image from the time mean of all the frames. This technique works because the data we are working with is at disk centre and over sufficient timescales such that solar rotation, satellite positional jitter and the point spread function of the instrument ensure that any true solar features are spread over a large area of the CCD. As a result nearest neighbour variation, as is selected by the discrete Laplacian only measures the variation of the CCD properties from pixel to pixel.

Having removed the dark frame, the proton impacts (which only affected 2 frames) were then removed by comparing frame differences. These operations greatly improved the images but there were still some significant artifacts, falling into two main categories: (a) isolated pixels that exhibited large, random variations with time; and (b) those that had a normal variation with time, but a temporal mean value significantly different to that of their neigbours.

Artificial features based on (a) were removed by looking at the temporal evolution of the standard deviation of the discrete Laplacian of each pixel. These pixels exhibited an abnormally high variation over the whole time 
period, often 10 times higher that that of their nearest neighbours, combined with unusual mean values. The data from these pixels was unrecoverable, and they were removed by replacing them with the average of their good neighbours.

Problematic pixels in category (b) did convey useful time evolution data, and can be explained by incorrect values in the supplied dark frames producing a DC offset in the data. These were optimised by keeping their time variation, but setting their mean to that of their nearest good neighbours.

The data was then derotated and interpolated with a small amount of spatial smoothing to match the MDI region of interest.

\subsection{Correlation Between X-Ray Bright points and magnetic CONVERGENCE}

In order to see if there is a causal relationship between the heating events and the flux removal, we need to show spatial and temporal alignment between them. As regions of flux removal occur when fluxes of opposite polarity are swept together by the supergranular flows, these areas will also represent a concentration of the magnetic field. In order to confirm that the heating is due to flux removal or submergence, rather than merely flux enhancement by migration, we need to be able to distinguish between these two effects.

Areas completely containing SXT brightenings at any point over the time range were selected. We used the criteria that any bright point had to exist for a minimum of two consecutive SXT frames, in order to distinguish it from random noise. Next the corresponding areas from the flux removal data and the magnetogram data were selected. The magnetogram data were smoothed to have the same spatial resolution as the flux removal data. The maximum values of the flux removal rate $\beta$ and the absolute flux density within the subimage were measured for each time frame, along with the maximum SXT intensity. The time evolution of 6 of these areas is presented in Figure 4, in which the SXT light curve for each region is given in the top panels, with the corresponding flux reduction rate $\beta$ and the absolute flux density in the middle and bottom panels respectively.

Region (i) has a large peak at start, corresponding to a very large rate of flux removal of $1.8 \times 10^{9} \mathrm{~Wb} \mathrm{~s}^{-1}$, the largest of the entire data set. There are two more peaks at around 22 and 26 hours, present in both the SXT data and the flux reduction rate, but not present in the absolute flux density plot. In region (ii) there is very low correlation between the SXT data and the flux removal rate. There is, however, a very strong correlation between the SXT data on regions (i) and (ii). This suggest that there is a magnetic structure linking these two regions that was disturbed by the flux removal event at region (i), causing a restructuring of the field, and consequent heating or 

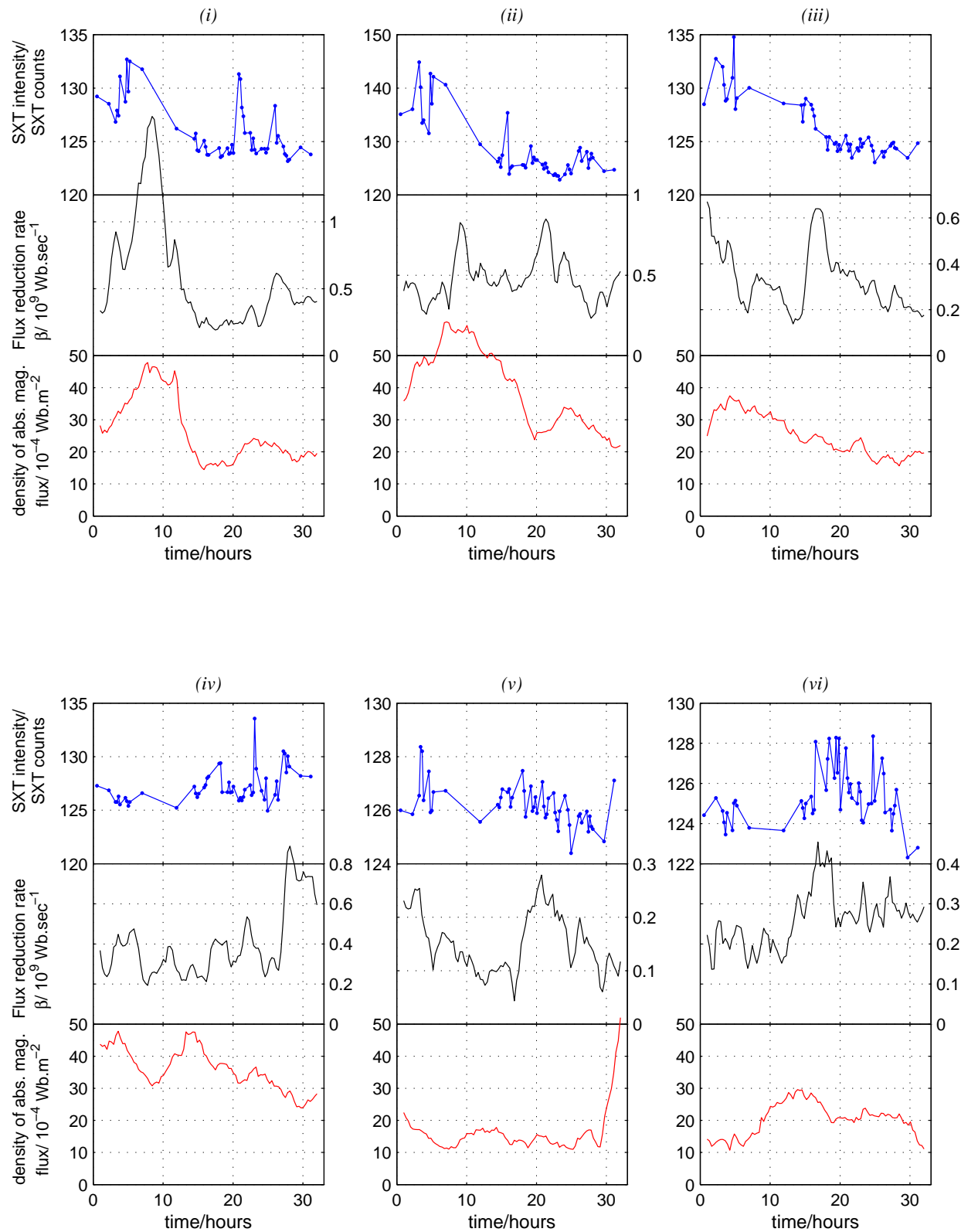

Figure 4. Time evolution of SXT emission intensity (top); $\beta$, the flux removal rate (middle) and the density of the absolute flux (bottom) for six selected regions in the quiet Sun. Note that the SXT coverage is discontinuous in time, with the points in the graphs representing the individual images. 



Figure 5. Spatial correlation between SXT brightenings and the rate of magnetic removal, $\beta$ for subsets of the full data set. Regions with high $\beta$ have rapid flux removal. The labels (i), (ii), (iii) and (vi) refer to the regions used in Figure 4. The contours follow the SXT data, and are included in all plots to guide the eye.

particle acceleration. The relative positions of regions (i), (ii), (iii) and (vi) are shown in Figure 5.

Regions (iii) and (iv) both show good correlation from around 15 hours onwards. Note the accurate time alignment of short timescale SXT brightenings and the flux removal rate.

Regions (v) and (vi) show strong relationships between the flux removal rate and the soft X-ray emission throughout the entire period. Again, notice the accurate matching of the timing of features between the two graphs, and the relatively poor correlation with the density of absolute flux throughout.

For all plots, the time alignment of the emission features between the soft $\mathrm{X}$-ray emission and the magnetic removal is good, much better than between the absolute magnetic flux and the SXT data. This indicates that the flux removal, rather than just the density of absolute flux, is causally linked to the X-ray emission. Note that the time coverage of SXT data is sparse, at less than $0.1 \%$ coverage, with gaps between consecutive frames ranging from a 
few minutes to several hours. Given the disparity in instrument cadence and coverage between SXT and MDI, it is not surprising that there are predicted flux evolution events from the MDI analysis that have no corresponding SXT signal.

These independent data sets, interpreted in the context of the results from Section 2 strongly suggest that the migration of magnetic elements under hydrodynamical flows leads to soft X-ray emission as the structures evolve.

We would expect heating due to submerging loops to be stochastic in nature (see next section); this combined with the sparse SXT coverage will result in the best correlations coming from regions in which the heating is a quasi-continuous series of small events.

Figure 5 shows the spatial correlation between SXT brightenings and the magnetic flux removal regions, underlining the causal link between disappearing flux and soft X-ray signals. Note that the algorithm used to produce these images is insensitive to flux emergence $(\beta<0)$, as at the point of emergence the field is predominantly horizontal, and therefore not visible on the disk centre, line-of-sight magnetograms used here. If the diameter of the boundary circle (shown in Figure 3) is increased, so that the vertical footpoint pairs of emerging loops can be contained, emerging loops can be detected, but the resolution and hence sensitivity to submerging structures, which are smaller, is reduced.

Combining the results in Figures 4 and 5 with the observations of (Harvey et al., 1999) and (Chae, Moon, and Pevtsov, 2004), which show that in the majority of cases flux is submerging at flux removal sites, then there must be a mechanism that connects submerging flux with heating events. We will address this explicitly in the next section.

\section{A heating mechanism for submerging loops}

The theoretical modelling of X-ray bright point appearance and the evolution of cancelling magnetic features has been addressed in many papers, including the seminal article by Priest, Parnell, and Martin (1994), and Longcope (1998) which suggest a magnetic reconnection mechanism giving rise to heating and X-ray emission. Other mechanisms have also been suggested, for example Brown et al., (2001) presents observations indicating a kink instability in a twisted flux loop, and more recently, Welsch (2006) which addresses the change in magnetic free energy in the corona as a result of flux cancellation.

There is also a considerable body of literature in which the changing geometry of large-scale flux tubes can be modelled in terms of time-dependent magnetic traps, suitable for flare evolution; for example, Somov and Ko- 


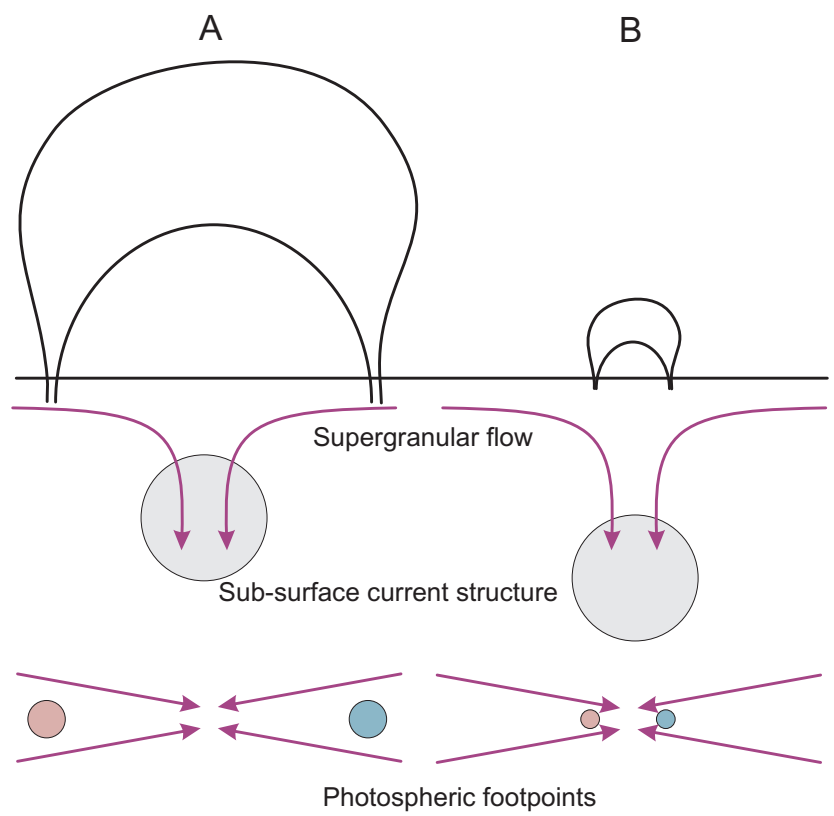

Figure 6. The geometry considered for particle acceleration, showing the loop-sustaining current structure being submerged by the downflow at a supergranular boundary, and the loop footpoints being drawn in by the surface flow, resulting in shrinking and submergence of the loop.

sugi (1997), Karlický and Kosugi (2004) and Giuliani, Neukirch, and Wood (2005). In this section we are confining our attention to small-scale features on the quiet Sun, and so our treatment here is less ambitious than the sophisticated models mentioned above, but is complementary to them in terms of suggesting another possible route for energy release.

Consider the evolution of the energy of a particle trapped in a magnetic mirror, the length of which is shortening. The context here is of small loops being dragged by the local fluid motion towards the edges of a convection cell, at which point there is a strong, downward directed fluid flow. It is reasonable to suppose that such downward flows are likely to drive deeper into the interior the sub-surface current-source of these loops. In the simplest possible model, as shown in Figure 6, we shall consider the case in which the geometry of the flux tube remains broadly unchanged but for a continuous sinking below the surface. This leads to a continuously reducing total path length between the mirroring regions which are associated with the narrowing of the flux tube at lower altitudes due to greater pressures there.

During the evolution of the flux tube, that is, its migration and submergence, it will retain a population of particles on average. All particles will 
be subjected to the motion inwards of the magnetic mirroring points, as the length of the tube above the photosphere reduces. At each interaction with these mirror points, particles will gain energy, acquiring a mirror velocity increment at each encounter. The lower energy component of the particle distribution (that is, below $10 \mathrm{eV}$ or so) will be heavily collisional, and so the heating effect from multiple encounters with the moving mirror points is very limited, since such particles are rapidly scattered into the loss cone, and are lost to the tube. Note that the definition of a loss-cone in curved geometry is more complex than in the linear magnetic mirror case, since the definition of parallel to the magnetic axis is only local. Although such particles are scattered out of the tube, other particles will diffuse in, in such a way that the tube integrity is maintained. For higher energy particles with energies of at least $100 \mathrm{eV}$, the collisional mean free path starts to approach the loop length (approximately 1\% of a $10 \mathrm{Mm}$ flux tube at $100 \mathrm{eV}$ ). Such particles are more likely to persist in the tube, given the decreasing collisional crosssection with increasing energy. Hence these particles are heated efficiently: they have more encounters with the moving mirrors, more frequently, and gain energy rapidly. At $1 \mathrm{keV}$, the collisional mean free path is $\sim 10 \mathrm{Mm}$, of the same order as the loop length, and so such particles will bounce many times. As a result, descending loops retain the hotter particles from the outset, accelerating them to energies where X-ray bright point emission occurs.

Notice that if the rate of loop shrinkage is constant, the heating rate increases as the loop size decreases, due to the shortening timescale between successive mirror encounters. The minimum size of loop for which this heating mechanism can work will be determined by a balance between the heating from the moving mirror regions and the losses due to the increasing collision rate as the loop sinks into the chromosphere. One consequence of this is that we would expect the heating to be 'bursty', as individual small loop structures reach their maximum heating rate. Note that this model does not rule out reconnection events. For converging magnetic elements that start far from each other to form a loop, earlier reconnection is likely; instead it gives an additional heating mechanism as the loop submerges.

\section{Conclusions}

In this article we have presented data that strongly suggest a ubiquitous small-scale heating mechanism driven by converging supergranular flows.

We have demonstrated that the photospheric velocity field is a good proxy for the motion of small magnetic elements in the quiet Sun. Using this relationship, and high resolution line-of-sight magnetograms, we have identified the regions in which magnetic field elements of opposite polarity 
are swept together and disappear. We find a clear spatial and temporal correlation between these field removal events and soft X-ray emission.

We have also presented a possible simple heating mechanism based on the heating of a shrinking loop by moving magnetic mirrors that is consistent with the behaviour of a small-scale magnetic structure being submerged by convergent supergranular flows.

As new instrumentation allows us to look in more detail at the Sun, it is clear that we will see ever increasing numbers of smaller events. There is some evidence that these small events may contribute significantly to coronal heating in the quiet Sun. In the near future, when the greatly increased resolution and cadence soft X-ray and vector magnetogram images from Hinode become available, we expect to be able to do a more detailed statistical study of this phenomenon, and quantify the energy so released.

\section{Acknowledgements}

We are grateful to PPARC for research funding. It is a pleasure to acknowledge very useful conversations with Lyndsay Fletcher.

\section{References}

Berger, T.E., Loefdahl, M.G., Shine, R.S., Title, A.M.: 1998, Measurements of Solar Magnetic Element Motion from High-Resolution Filtergrams. Astrophys. J. 495, 973-+. doi:10.1086/305309.

Brown, D.S., Parnell, C.E., Deluca, E.E., Golub, L., McMullen, R.A.: 2001, The Magnetic Structure of a Coronal X-Ray Bright Point. Solar Phys. 201, 305-321. doi:10.1023/A:1017907406350.

Chae, J., Moon, Y.J., Pevtsov, A.A.: 2004, Observational Evidence of Magnetic Flux Submergence in Flux Cancellation Sites. Astrophys. J. Lett. 602, L65-L68. doi:10.1086/382222.

Chae, J., Moon, Y.J., Wang, H., Yun, H.S.: 2002, Flux Cancellation Rates and Converging Speeds of Canceling Magnetic Features. Solar Phys. 207, 73-85.

DeRosa, M.L., Toomre, J.: 2004, Evolution of Solar Supergranulation. Astrophys. J. 616, 1242 - 1260. doi:10.1086/424920.

Erdélyi, R.: 2005, Heating of the Solar Corona: Review. Publications of the Astronomy Deparment of the Eotvos Lorand University 15, 7-+.

Giuliani, P., Neukirch, T., Wood, P.: 2005, Particle Motion in Collapsing Magnetic Traps in Solar Flares. I. Kinematic Theory of Collapsing Magnetic Traps. Astrophys. J. 635, 636-646. doi:10.1086/497366.

Hagenaar, H.J., Schrijver, C.J., Title, A.M., Shine, R.A.: 1999, Dispersal of Magnetic Flux in the Quiet Solar Photosphere. Astrophys. J. 511, 932-944. doi:10.1086/306691.

Harvey, K.L., Jones, H.P., Schrijver, C.J., Penn, M.J.: 1999, Does Magnetic Flux Submerge at Flux Cancelation Sites? Solar Phys. 190, 35-44. doi:10.1023/A:1005237719407.

Hudson, H.S.: 1991, Solar flares, microflares, nanoflares, and coronal heating. Solar Phys. 133, $357-369$. 
Karlický, M., Kosugi, T.: 2004, Acceleration and heating processes in a collapsing magnetic trap. Astron. Astrophys. 419, 1159-1168. doi:10.1051/0004-6361:20034323.

Lisle, J., De Rosa, M., Toomre, J.: 2000, New Approach to Study Extended Evolution of Supergranular Flows and Their Advection of Magnetic Elements. Solar Phys. 197, $21-30$.

Longcope, D.W.: 1998, A Model for Current Sheets and Reconnection in X-Ray Bright Points. Astrophys. J. 507, 433-442. doi:10.1086/306319.

Lui, Y., Norton, A.A.: SOI Technical note 01-144, Mdi measurement errors: The magnetic perspective. http://soi.stanford.edu/technotes/01.144/TN01-144.pdf.

Parnell, C.E., Jupp, P.E.: 2000, Statistical Analysis of the Energy Distribution of Nanoflares in the Quiet Sun. Astrophys. J. 529, 554-569. doi:10.1086/308271.

Potts, H.E.: 2007, Supergranulation movie. http://www.astro.gla.ac.uk/users/hugh/ supergranulation/supergran_mag_overlay.avi.

Potts, H.E., Barrett, R.K., Diver, D.A.: 2003, Reduction of interpolation errors when using local correlation tracking for motion detection. Solar Phys. 217, 69-78.

Potts, H.E., Barrett, R.K., Diver, D.A.: 2004, Balltracking: An highly efficient method for tracking flow fields. Astron. Astrophys. 424, 253-262. doi:10.1051/00046361:20035891.

Potts, H.E., Diver, D.A.: 2007, Automatic recognition and characterisation of supergranular cells from photospheric velocity fields. Solar Phys., in press.

Priest, E.R., Parnell, C.E., Martin, S.F.: 1994, A converging flux model of an X-ray bright point and an associated canceling magnetic feature. Astrophys. J. 427, 459-474. doi:10.1086/174157.

Scherrer, P.H., Bogart, R.S., Bush, R.I., Hoeksema, J.T., Kosovichev, A.G., Schou, J., Rosenberg, W., Springer, L., Tarbell, T.D., Title, A., Wolfson, C.J., Zayer, I., MDI Engineering Team, : 1995, The Solar Oscillations Investigation - Michelson Doppler Imager. Solar Phys. 162, 129-188.

Simon, G.W., Title, A.M., Topka, K.P., Tarbell, T.D., Shine, R.A., Ferguson, S.H., Zirin, H., SOUP Team, : 1988, On the relation between photospheric flow fields and the magnetic field distribution on the solar surface. Astrophys. J. 327, 964-967. doi:10.1086/166253.

Somov, B.V., Kosugi, T.: 1997, Collisionless Reconnection and High-Energy Particle Acceleration in Solar Flares. Astrophys. J. 485, 859-+. doi:10.1086/304449.

Webb, D.F., Martin, S.F., Moses, D., Harvey, J.W.: 1993, The correspondence between $\mathrm{X}$-ray bright points and evolving magnetic features in the quiet sun. Solar Phys. 144, $15-35$.

Welsch, B.T.: 2006, Magnetic Flux Cancellation and Coronal Magnetic Energy. Astrophys. J. 638, 1101-1109. doi:10.1086/498638. 